\title{
MEAN CONVERGENCE FOR INTERMEDIATELY TRIMMED BIRKHOFF SUMS OF OBSERVABLES WITH REGULARLY VARYING TAILS
}

\author{
MARC KESSEBÖHMER AND TANJA SCHINDLER
}

\begin{abstract}
On a measure theoretical dynamical system with spectral gap property we consider non-integrable observables with regularly varying tails and fulfilling a mild mixing condition. We show that the normed trimmed sum process of these observables then converges in mean. This result is new also for the special case of i.i.d. random variables and contrasts the general case where mean convergence might fail even though a strong law of large numbers holds. To illuminate the required mixing condition we give an explicit example of a dynamical system fulfilling a spectral gap property and an observable with regularly varying tails but without the assumed mixing condition such that mean convergence fails.
\end{abstract}

\section{IntroduCtion AND STATEMENT OF MAIN RESUltS}

We consider an ergodic measure preserving dynamical system $(\Omega, \mathcal{A}, T, \mu)$ with $\mu$ a probability measure and stochastic processes given by the Birkhoff sums $\mathrm{S}_{n} \chi:=\sum_{k=1}^{n} \chi \circ T^{k-1}, n \in \mathbb{N}$, with $\mathrm{S}_{0} \chi=0$ for some function $\chi \in \mathcal{M}(\mathcal{A})^{+}:=\left\{f: \Omega \rightarrow \mathbb{R}_{>0}: f\right.$ is $\mathcal{A}$-measurable $\}$ sometimes called observable. If $\int \chi \mathrm{d} \mu$ is finite, then we obtain by Birkhoff's ergodic theorem-combining pointwise and mean convergence - that $\mu$-almost surely (a.s.)

$$
\lim _{n \rightarrow \infty} \frac{\mathrm{S}_{n} \chi}{\int \mathrm{S}_{n} \chi \mathrm{d} \mu}=1
$$

i.e. the strong law of large numbers is fulfilled with norming sequence $\left(\int S_{n} \chi d \mu\right)$, whereas in the case $\int \chi \mathrm{d} \mu=\infty$, Aaronson ruled out the possibility of a strong law of large numbers no matter which norming sequence we choose, see Aar77. However, in certain cases after deleting a number of the largest summands from the partial $n$-sums a strong law of large numbers holds. More precisely, for each $n \in \mathbb{N}$ we choose a permutation $\sigma \in \mathcal{S}_{n}$ of $\{0, \ldots, n-1\}$ with $\chi \circ T^{\sigma(1)} \geq$ $\chi \circ T^{\sigma(2)} \geq \ldots \geq \chi \circ T^{\sigma(n)}$ and for given $b_{n} \in \mathbb{N}_{0}$ we define

$$
\mathrm{S}_{n}^{b_{n}} \chi:=\sum_{k=b_{n}}^{n-1} \chi \circ T^{\sigma(k)} \text {. }
$$

If $b_{n}=r \in \mathbb{N}$ is fixed for all $n \in \mathbb{N}$, then $\left(\mathrm{S}_{n}^{r} \varphi\right)$ is called a lightly trimmed sum process. If we allow the sequence $\left(b_{n}\right) \in \mathbb{N}^{\mathbb{N}}$ to diverge to infinity such that $b_{n}=o(n)$, i.e. $\lim _{n \rightarrow \infty} b_{n} / n=0$, then $\left(\mathrm{S}_{n}^{b_{n}} \varphi\right)$ is called an intermediately (also moderately) trimmed sum process.

The special case of regularly varying tail variables with index strictly between -1 and 0 has been considered by the authors in $\mathrm{KS18}$. That is for $F: x \mapsto \mu(\chi \leq x)$ denoting the distribution function of $\chi$ we require that $1-F(x)=x^{-\alpha} L(x)$ with $0<\alpha<1$ and $L$ a slowly varying function, i.e. for every $c>0$ we have $L(c x) \sim L(x)$. Here, $u(x) \sim w(x)$ means that $u$ is asymptotic to $w$ at infinity, i.e. $\lim _{x \rightarrow \infty} u(x) / w(x)=1$.

Date: March 25, 2019.

2010 Mathematics Subject Classification. Primary: 60F25 Secondary: 37A05, 37A30, 37A25, 60G10.

Key words and phrases. mean convergence, trimmed sum process, transfer operator, spectral method, $\psi$-mixing, piecewise expanding interval maps. 
Under certain properties of the underlying process to be discussed later an intermediately trimmed strong law has been proven for such observables, i.e. there exist a non-negative integer sequence $\left(b_{n}\right)$ tending to infinity with $b_{n}=o(n)$ and a norming sequence $\left(d_{n}\right)$ such that

$$
\lim _{n \rightarrow \infty} \frac{\mathrm{S}_{n}^{b_{n}} \chi}{d_{n}}=1 \text { a.s. }
$$

Additionally, an asymptotic formula for $\left(d_{n}\right)$ depending on $\left(b_{n}\right)$ has been provided in $\mathrm{KS} 18$, Theorem 1.7]. The condition under which these trimming results hold are in particular a spectral gap property for the transfer operator und some regularity conditions on the observable $\chi$, the precise conditions are stated as Property $\mathfrak{D}$ in Definition 1.3 .

The above stated intermediately trimmed strong law can be seen as an analog to Birkhoff's ergodic theorem. However in the finite case, Birkhoff's ergodic theorem also implies that the norming sequence $\left(d_{n}\right)$ can be chosen as $\left(\int \mathrm{S}_{n} \chi \mathrm{d} \mu\right)$, see e.g. [KMS16, Prop. 2.4.21]. It is the purpose of the present paper to show that for regularly varying tail distributions with exponent strictly between -1 and 0 we also have $d_{n} \sim \int \mathrm{S}_{n}^{b_{n}} \mathrm{~d} \mu$ and in this way to give an analog statement of the ergodic theorem for the trimmed sum process. Since in Lemma 2.2 we also show convergence in probability, the above asymptotic is in fact equivalent to mean convergence (Theorem 1.5) and gives thus also an analog for von Neumann's $\mathcal{L}^{1}$ ergodic theorem. Crucial for our analysis will be an additional condition on $\left(\chi \circ T^{n-1}\right)$ in terms of the $\boldsymbol{\psi}$-mixing coefficients introduced in Definition 1.4. We will show that Property $\mathfrak{D}$ alone is indeed not strong enough for our main results to hold by providing an example with large $\boldsymbol{\psi}$-mixing coefficients and which can not obey any mean convergence, as $\int \mathrm{S}_{n}^{b_{n}} \mathrm{~d} \mu=\infty$, for all $n \in \mathbb{N}$ and any reasonable trimming sequence $\left(b_{n}\right)$, see Theorem 1.10 .

In the case of general distribution functions and the same mixing conditions it is too much to hope for such a mean convergence under trimming. The authors of this paper gave an example in [KS17, Remark 3] for i.i.d. random variables for which an intermediately trimmed strong law holds but $\int \mathrm{S}_{n}^{b_{n}} \mathrm{~d} \mu=\infty$, for all $n \in \mathbb{N}$.

It is also worth mentioning that the almost sure trimming results mentioned above have predecessors in results for i.i.d. random variables where a vast literature for trimming results both for weak as well as for strong limit theorems exists. However, to the author's knowledge the result given in this paper has not been proven for i.i.d. random variables either. We will here only give an overview of a number of strong convergence results. First of all one realizes that also for i.i.d. random variables a lightly trimmed strong law can not hold for random variables with regularly varying tail with exponent strictly between -1 and 0 . By a lightly trimmed strong law we mean the existence of $r \in \mathbb{N}$ and a sequence $\left(d_{n}\right)$ of positive reals such that $\lim _{n \rightarrow \infty} S_{n}^{r} \chi / d_{n}=1$ a.s. This can be deduced from the fact that there is no weak law of large numbers for random variables with such a distribution function, see [Fel71, VII.7 Theorem 2 and VIII.9 Theorem 1] and a result by Kesten which states that light trimming has no influence on weak convergence, see Kes93. However, an intermediately trimmed strong law in the i.i.d. case can be deduced from results by Haeusler and Mason, see [HM87] and [Hae93]. They proved generalized laws of the iterated logarithm under trimming from which an intermediately trimmed strong law follows and one can also infer a lower bound for $\left(b_{n}\right)$. Indeed, this lower bound coincides with the lower bound for $\left(b_{n}\right)$ in the dynamical systems case given in [KS18]. The examples for which the setting of [KS18 holds are e.g. piecewise expanding interval maps and subshifts of finite type as shown in [KS18] and KS19] respectively.

Some other trimming results have also been generalized to different dynamical system settings where $\boldsymbol{\psi}$-mixing also plays an important role. In fact, Aaronson and Nakada showed in AN03. a lightly trimmed strong law for $\boldsymbol{\psi}$-mixing random variables which have particular distribution functions. This result generalizes the results of Mori for the i.i.d. case, see [Mor76, Mor77]. Aaronson and Nakada also gave an example of a non $\boldsymbol{\psi}$-mixing process with the same distribution function not fulfilling a lightly trimmed strong law. Furthermore, Haynes gave in Hay14 a quantitative generalization for $\boldsymbol{\psi}$-mixing random variables of a result by Diamond and Vaaler who showed a lightly trimmed strong law for the continued fraction digits, see [DV86]. Haynes also 
compared this result with an observable on the doubling map for which the system is strongly mixing but not $\boldsymbol{\psi}$-mixing and for which a lightly trimmed strong laws fails to hold. This example also fulfills the spectral gap property and Property $\mathfrak{D}$.

The results of this paper rely on two main properties: First, on an exponential inequality for dynamical systems which fulfill a spectral gap property with respect to the transfer operator, and second, on the $\boldsymbol{\psi}$-mixing property. The proof of the exponential inequality given in [KS18] is similar to the Nagaev-Guivarc'h spectral method for the central limit theorem. The spectral gap property for dynamical systems is a typical assumption under which limit theorems for dynamical systems can be proven, see the review papers [Gou15] and [FJ03] and references therein for further information and applications of the transfer operator method as well as examples of dynamical systems fulfilling a spectral gap property.

During the last decade there has also been some significant interest in other limit theorems for dynamical systems with heavy tailed distributions using transfer operator techniques, particularly convergence to a stable law, see the paper by Aaronson and Denker, AD01, for sufficient and the paper by Gouëzel, Gou10, for necessary conditions and previous results by Sarig, Sar06. Furthermore, see also the generalization by Melbourne and Zweimüller to intermittent maps, [MZ15], and by Tyran-Kaminska to a functional convergence, see [TK10]. The additional condition of $\boldsymbol{\psi}$-mixing is often necessary for proving limit theorems as illustrated above for the trimmed strong laws. Some results have also been proven under the combined assumptions of a spectral gap property and $\boldsymbol{\psi}$-mixing, as for example the law of an iterated logarithm for non-integrable random variables by Aaronson and Zweimüller, AZ14.

In Example 1.9 we will give conditions on piecewise expanding interval maps and on the observable $\chi$ such that Property $\mathfrak{D}$ as well as the $\boldsymbol{\psi}$-mixing condition are fulfilled.

1.1. Basic setting. First we will make the notion of spectral gap precise and then restate the two crucial properties from [KS18]. The first, Property $\mathfrak{C}$, considers dynamical systems with a spectral gap property. Afterwards we define our main property, Property $\mathfrak{D}$, for which different convergence theorems have been proven in [KS18] and under which we will prove a mean convergence theorem under trimming.

Definition 1.1 (Spectral gap). Suppose $\mathcal{F}$ is a Banach space and $U: \mathcal{F} \rightarrow \mathcal{F}$ a bounded linear operator. We say that $U$ has a spectral gap if there exists a decomposition $U=\lambda P+N$ with $\lambda \in \mathbb{C}$ and $P, N$ bounded linear operators such that

- $P$ is a one-dimensional projection, i.e. $P^{2}=P$ and its image is one-dimensional,

- $N$ is such that $\rho(N)<|\lambda|$, where $\rho$ denotes the spectral radius,

- $P$ and $N$ are orthogonal, i.e. $P N=N P=0$.

Definition 1.2 (Property $\mathfrak{C}$, [KS18, Definition 1.1]). Let $(\Omega, \mathcal{A}, T, \mu)$ be a dynamical system with $T$ a non-singular transformation and $\widehat{T}: \mathcal{L}^{1} \rightarrow \mathcal{L}^{1}$ be the transfer operator of $T$, i.e. the uniquely defined operator such that for all $f \in \mathcal{L}^{1}$ and $g \in \mathcal{L}^{\infty}$ we have

$$
\int \widehat{T} f \cdot g \mathrm{~d} \mu=\int f \cdot g \circ T \mathrm{~d} \mu,
$$

see e.g. KMS16, Section 2.3] for further details. Furthermore, let $\mathcal{F}$ be subset of the measurable functions forming a Banach algebra with respect to the norm $\|\cdot\|$. We say that $(\Omega, \mathcal{A}, T, \mu, \mathcal{F},\|\cdot\|)$ has Property $\mathfrak{C}$ if the following conditions hold:

- $\mu$ is a $T$-invariant, mixing probability measure.

- $\mathcal{F}$ contains the constant functions and for all $f \in \mathcal{F}$ we have

$$
\|f\| \geq|f|_{\infty} .
$$


- $\widehat{T}$ is a bounded linear operator with respect to $\|\cdot\|$, i.e. there exists a constant $K_{0}>0$ such that for all $f \in \mathcal{F}$ we have

$$
\|\widehat{T} f\| \leq K_{0} \cdot\|f\| .
$$

- $\widehat{T}$ acting on the Banach space $\mathcal{F}$ with norm $\|\cdot\|$ has a spectral gap.

The above mentioned property is a widely used setting for dynamical systems. In particular it implies that the transfer operator has 1 as a unique and simple eigenvalue on the unit circle and that an exponential decay of correlation is guaranteed.

However, in order to state our main theorems we need additional assumptions on the observable $\chi$ defined on a system fulfilling Property $\mathfrak{C}$.

Definition 1.3 (Property $\mathfrak{D}$, KS18, Definition 1.2]). We say that $(\Omega, \mathcal{A}, T, \mu, \mathcal{F},\|\cdot\|, \chi)$ has Property $\mathfrak{D}$ if the following conditions hold:

- $(\Omega, \mathcal{A}, T, \mu, \mathcal{F},\|\cdot\|)$ fulfills Property $\mathfrak{C}$.

- $\chi \in \mathcal{M}(\mathcal{A})^{+}$and with ${ }^{\ell} \chi:=\chi \cdot \mathbb{1}_{\{\chi \leq \ell\}}$ there exists $K_{1}>0$ such that for all $\ell \in \mathbb{R}_{\geq 0}$,

$$
\|\ell \chi\| \leq K_{1} \cdot \ell \quad \text { and } \quad\left\|\mathbb{1}_{\{\chi>\ell\}}\right\| \leq K_{1} .
$$

Finally, to state our main theorem we give the precise definition of $\boldsymbol{\psi}$-mixing following [Bra05]. Note that in the literature there are sometimes subtle differences defining this notion.

Definition 1.4. Let $(\Omega, \mathcal{A}, \mathbb{P})$ be a probability measure space and $\mathcal{B}, \mathcal{C} \subset \mathcal{A}$ two $\sigma$-fields, then the following measure of dependence is defined

$$
\boldsymbol{\psi}(\mathcal{B}, \mathcal{C}):=\sup \left\{\left|\frac{\mathbb{P}(B \cap C)}{\mathbb{P}(B) \cdot \mathbb{P}(C)}-1\right|: B \in \mathcal{B}, C \in \mathcal{C}, \mathbb{P}(B), \mathbb{P}(C)>0\right\} .
$$

Furthermore, let $\left(X_{n}\right)_{n \in \mathbb{N}}$ be a (not necessarily stationary) sequence of random variables. For $-\infty \leq J \leq L \leq \infty$ we can define a $\sigma$-field by

$$
\mathcal{A}_{J}^{L}:=\sigma\left(X_{k}, J \leq k \leq L, k \in \mathbb{Z}\right) .
$$

With this at hand the $\boldsymbol{\psi}$-mixing coefficients are defined by

$$
\psi(n):=\sup _{k \in \mathbb{N}} \psi\left(\mathcal{A}_{-\infty}^{k}, \mathcal{A}_{k+n}^{\infty}\right) .
$$

The sequence of random variables $\left(X_{n}\right)$ is said to be $\boldsymbol{\psi}$-mixing if $\lim _{n \rightarrow \infty} \boldsymbol{\psi}(n)=0$.

1.2. Main results. For $L$ being slowly varying we denote by $L^{\#}$ a de Bruijn conjugate of $L$, i.e. a slowly varying function satisfying

$$
\lim _{x \rightarrow \infty} L(x) \cdot L^{\#}(x L(x))=1=\lim _{x \rightarrow \infty} L^{\#}(x) \cdot L\left(x L^{\#}(x)\right) .
$$

For more details see [BGT87, Section 1.5.7 and Appendix 5]. Then our first main result reads as follows.

Theorem 1.5. Let $(\Omega, \mathcal{A}, T, \mu, \mathcal{F},\|\cdot\|, \chi)$ fulfill Property $\mathfrak{D}$ and assume that $\mu(\chi>x)=L(x) / x^{\alpha}$, where $L$ is a slowly varying function and $0<\alpha<1$. Further, let $\left(b_{n}\right)$ be a sequence of natural numbers tending to infinity such that $b_{n}=o(n)$. We assume that at least one $\boldsymbol{\psi}$-mixing coefficient of the sequence of random variables $\left(\chi \circ T^{n-1}\right)_{n \in \mathbb{N}}$ is strictly less than one. Then mean convergence holds with norming sequence

$$
d_{n}:=\frac{\alpha}{1-\alpha} \cdot n^{1 / \alpha} \cdot b_{n}^{1-1 / \alpha} \cdot\left(L^{-1 / \alpha}\right)^{\#}\left(\left(\frac{n}{b_{n}}\right)^{1 / \alpha}\right),
$$

that is

$$
\lim _{n \rightarrow \infty} \int\left|\frac{S_{n}^{b_{n}} \chi}{d_{n}}-1\right| \mathrm{d} \mu=0
$$


Remark 1.6. It has been shown in [KS18] that under Property $\mathfrak{D}$ and with $\left(b_{n}\right)$ growing sufficiently fast we have $\lim _{n \rightarrow \infty} \mathrm{S}_{n}^{b_{n}} \chi / d_{n}=1$ a.s. where $\left(d_{n}\right)$ shows the same asymptotic as (5). Combining this statement with Theorem 1.5 yields that for sufficiently fast growing $\left(b_{n}\right)$ we have

$$
\lim _{n \rightarrow \infty} \frac{\mathrm{S}_{n}^{b_{n}} \chi}{\int \mathrm{S}_{n}^{b_{n}} \chi \mathrm{d} \mu}=1 \text { a.s. }
$$

Remark 1.7. Note that $\boldsymbol{\psi}$-mixing is sufficient but not necessary for Theorem 1.5

For our main example, we first define the space of functions of bounded variation. For simplicity, we restrict ourself to the interval $[0,1]$ and let $\mathcal{B}$ denote the Borel sets of $[0,1]$.

Definition 1.8. Let $\varphi:[0,1] \rightarrow \mathbb{R}$. Then the variation $\operatorname{var}(\varphi)$ of $\varphi$ is given by

$$
\operatorname{var}(\varphi):=\sup \left\{\sum_{i=1}^{n}\left|\varphi\left(x_{i}\right)-\varphi\left(x_{i-1}\right)\right|: n \geq 1, x_{i} \in[0,1], x_{0}<x_{1}<\ldots<x_{n}\right\}
$$

and we define

$$
\mathrm{V}(\varphi):=\inf \left\{\operatorname{var}\left(\varphi^{\prime}\right): \varphi^{\prime} \text { is a version of } \varphi\right\}
$$

By $B V$ we denote the Banach space of functions of bounded variation, i.e. of functions $\varphi$ fulfilling $\mathrm{V}(\varphi)<\infty$. It is equipped with the norm $\|\varphi\|_{B V}:=|\varphi|_{\infty}+\mathrm{V}(\varphi)$.

With this we can state our main example.

Example 1.9. Let $\Omega^{\prime} \subset[0,1]$ be a dense and open set such that $\mu\left(\Omega^{\prime}\right)=1$ and let $\mathcal{I}:=\left(I_{n}\right)_{n \in \mathbb{N}}$ be a countable family of closed intervals with disjoint interiors and for any $I_{n}$ such that the set $I_{n} \cap\left([0,1] \backslash \Omega^{\prime}\right)$ consists exactly of the endpoints of $I_{n}$. Furthermore, we assume that $T$ fulfills the following properties:

- (Adler's condition) $T_{n}:=\left.T\right|_{I_{n}} \in \mathcal{C}^{2}$ and $T^{\prime \prime} /\left(T^{\prime}\right)^{2}$ is bounded on $\Omega^{\prime}$.

- (Finite image condition) $\#\left\{T I_{n}: I_{n} \in \mathcal{I}\right\}<\infty$.

- (Uniform expansion) There exists $m>1$ such that $\left|T_{n}^{\prime}\right| \geq m$ for all $n \in \mathbb{N}$.

- $T$ is topologically mixing.

Furthermore, we assume that $\chi$ is constant on the interior of each interval $I_{n}$ and there exists a constant $k>0$ such that for all $\ell \in \mathbb{R}_{\geq 0}$

$$
\mathrm{V}\left({ }^{\ell} \chi\right) \leq k \cdot \ell \quad \text { and } \quad \mathrm{V}\left(\mathbb{1}_{\{\chi>\ell\}}\right) \leq k .
$$

Then there exists a probability measure $\mu$ absolutely continuous to the Lebesgue measure such that $\left([0,1], \mathcal{B}, T, \mu, B V,\|\cdot\|_{B V}, \chi\right)$ fulfills Property $\mathfrak{D}$ and $\left(\chi \circ T^{n-1}\right)$ is a $\psi$-mixing.

We note here that this example mainly relies on results in Ryc83 on piecewise expanding interval maps on countable partitions generalizing [LY73] where finite partitions are considered. It follows from [Zwe98] that $\left([0,1], \mathcal{B}, T, \mu, B V,\|\cdot\|_{B V}\right)$ fulfills Property $\mathfrak{C}$. Furthermore, (6) implies (4) and thus $\left([0,1], \mathcal{B}, T, \mu, B V,\|\cdot\|_{B V}\right)$ fulfills Property $\mathfrak{D}$. This was discussed in detail in KS18, Section 1.4]. Finally, that $\left(\chi \circ T^{n-1}\right)$ is $\boldsymbol{\psi}$-mixing follows by [AN05, Theorem 1]. As an explicit example one could choose the partition $I_{n}:=[1 /(n+1), 1 / n], n \in \mathbb{N}$, with any $T$ fulfilling the above properties with respect to this partition and $\chi=\sum_{n \in \mathbb{N}} n^{1 / \alpha} \cdot \mathbb{1}_{I_{n}}$ and the underlying invariant measure calculated as in Ryc83.

Our next theorem shows that indeed Property $\mathfrak{D}$ alone is not sufficient for our main theorem.

Theorem 1.10. Let $\Omega:=[0,1)$ and $T:=2 x \bmod 1$ with $\mu$ the Lebesgue measure restricted to $[0,1)$. Further define $\chi:[0,1) \rightarrow \mathbb{R}_{>0}$ by $\chi(x)=x^{-\gamma}$ with $\gamma>1$. Then there exists a Banach 
space $\mathcal{F}$ with a norm $\|\cdot\|$ such that $([0,1), \mathcal{B}, T, \mu, \mathcal{F},\|\cdot\|, \chi)$ fulfills Property $\mathfrak{D}$. If on the other hand $\left(b_{n}\right)$ tends to infinity with $b_{n}=o(n)$, then

$$
\int \mathrm{S}_{n}^{b_{n}} \chi \mathrm{d} \mu=\infty
$$

for all $n \in \mathbb{N}$.

Remark 1.11. To the authors' knowledge this theorem is also a new result for the setting of i.i.d. random variables. Indeed the case of i.i.d. random variables follows as a special case from this setting. A proof of this fact will be given in Section 2.3 .

\section{Proofs of MAin theorems}

The second part of our paper is devoted to the proofs of the theorems; the proof of Theorem 1.5 will be given in Section 2.1, the proof of Theorem 1.10 in Section 2.2, and the proof of Remark 1.11 in Section 2.3.

2.1. Proof of Theorem 1.5. Theorem $[1.5$ is proven by proving the following two lemmas:

Lemma 2.1. Let $(\Omega, \mathcal{A}, T, \mu, \mathcal{F},\|\cdot\|, \chi)$ fulfill Property $\mathfrak{D}$ and assume that $\mu(\chi>x)=L(x) / x^{\alpha}$, where $L$ is a slowly varying function and $0<\alpha<1$. Further, let $\left(b_{n}\right)$ be a sequence of natural numbers tending to infinity such that $b_{n}=o(n)$. We assume that at least one $\boldsymbol{\psi}$-mixing coefficient of the sequence of random variables $\left(\chi \circ T^{n-1}\right)_{n \in \mathbb{N}}$ is strictly less than one. Then $\int S_{n}^{b_{n}} \chi \sim d_{n}$ with $\left(d_{n}\right)$ as in (5).

Lemma 2.2. Let $(\Omega, \mathcal{A}, T, \mu, \mathcal{F},\|\cdot\|, \chi)$ fulfill Property $\mathfrak{D}$ and assume that $\mu(\chi>x)=L(x) / x^{\alpha}$, where $L$ is a slowly varying function and $0<\alpha<1$. Further, let $\left(b_{n}\right)$ be a sequence of natural numbers tending to infinity such that $b_{n}=o(n)$. Then with $\left(d_{n}\right)$ given in (5), we have the following convergence in probability:

$$
\lim _{n \rightarrow \infty} \mu\left(\left|\frac{S_{n}^{b_{n}} \chi}{d_{n}}-1\right|>\epsilon\right)=0, \text { for all } \epsilon>0 .
$$

Using Pratt's theorem [Pra60] in combination with Lemma 2.1 and 2.2 immediately gives the statement of Theorem 1.5 .

In preparation of the proof of these lemmas, for $\chi: \Omega \rightarrow \mathbb{R}_{\geq 0}$ and a real valued sequence $\left(f_{n}\right)_{n \in \mathbb{N}}$ we recall the definition of the truncated function

$$
{ }^{f_{n}} \chi:=\chi \cdot \mathbb{1}_{\left\{\chi \leq f_{n}\right\}}
$$

given in Definition 1.3 and define the associated truncated sum

$$
\mathbf{T}_{n}^{f_{n}} \chi:=\sum_{k=1}^{n} f_{n} \chi \circ T^{k-1}
$$

If $f_{n}$ tends to infinity, we have that

$$
\int \mathrm{T}_{n}^{f_{n}} \chi \mathrm{d} \mu \sim n \cdot \frac{\alpha}{1-\alpha} \cdot L\left(f_{n}\right) \cdot f_{n}^{1-\alpha},
$$

see [KS18, Lemma 3.18] for a detailed calculation.

Proof of Lemma 2.1. We recall that $F$ is the distribution function of $\chi$ with respect to $\mu$, i.e. $F(x)=\mu(\chi \leq x)=1-L(x) / x^{\alpha}$. Let $\left(\zeta_{n}\right)$ be defined as $\zeta_{n}:=b_{n}^{2 / 3}$ and set

$$
g_{n}:=F^{\leftarrow}\left(1-\frac{b_{n}-\zeta_{n}}{n}\right) .
$$

We will split the proof of the lemma into the following parts: 
(A) We have that

$$
\int \mathrm{T}_{n}^{g_{n}} \chi \mathrm{d} \mu \sim \frac{\alpha}{1-\alpha} \cdot n^{1 / \alpha} \cdot b_{n}^{1-1 / \alpha} \cdot\left(L^{1 / \alpha}\right)^{\#}\left(\left(\frac{n}{b_{n}}\right)^{1 / \alpha}\right) .
$$

(B) For all $\epsilon>0$ there exists $N \in \mathbb{N}$ such that for all $n \geq N$

$$
\int \mathrm{S}_{n}^{b_{n}} \chi \mathrm{d} \mu \leq(1+\epsilon) \int \mathrm{T}_{n}^{g_{n}} \chi \mathrm{d} \mu .
$$

(C) For all $\epsilon>0$ there exists $N \in \mathbb{N}$ such that for all $n \geq N$

$$
\int \mathrm{S}_{n}^{b_{n}} \chi \mathrm{d} \mu \geq(1-\epsilon) \int \mathrm{T}_{n}^{g_{n}} \chi \mathrm{d} \mu \text {. }
$$

Proof of $(A)$ The proof of a statement similar to (A) can be found at the end of the proof of Theorem 1.7 in [KS18. Indeed, there it is shown that $\int \mathrm{T}_{n}^{f_{n}} \chi \mathrm{d} \mu$ is asymptotic to the right hand side of (9). The sequence $\left(f_{n}\right)$ does not necessarily coincide with $\left(g_{n}\right)$. However, the sequences can be written as $f_{n}=F^{\leftarrow}\left(1-v_{n} / n\right)$ and $g_{n}=F^{\leftarrow}\left(1-w_{n} / n\right)$ with $v_{n} \sim u_{n} \sim b_{n}$. The proof in [KS18] essentially proves that $\int \mathrm{T}_{n}^{f_{n}} \chi \mathrm{d} \mu \sim \alpha /(1-\alpha) \cdot n^{1 / \alpha} \cdot v_{n}^{1-1 / \alpha} \cdot\left(L^{-1 / \alpha}\right)^{\#}\left(\left(n / v_{n}\right)^{1 / \alpha}\right)$ allowing us to conclude from $v_{n} \sim b_{n}$ the asymptotic in (9). The sequence $\left(g_{n}\right)$ can be treated analogously.

Proof of $(B)$. In order to prove (B) we set for $k, n \in \mathbb{N}$,

$$
\begin{aligned}
\Gamma_{n} & :=\left\{\mathrm{S}_{n}^{b_{n}} \chi \leq \mathrm{T}_{n}^{g_{n}} \chi\right\}, \\
\Delta_{k, n} & :=\left\{\mathrm{T}_{n}^{2^{k-1} \cdot g_{n}} \chi<\mathrm{S}_{n}^{b_{n}} \chi \leq \mathrm{T}_{n}^{2^{k} \cdot g_{n}} \chi\right\}, \\
\Phi_{k, n} & :=\left\{\mathrm{T}_{n}^{2^{k-1} \cdot g_{n}} \chi<\mathrm{S}_{n}^{b_{n}} \chi\right\} .
\end{aligned}
$$

Clearly,

$$
\begin{aligned}
\int \mathrm{S}_{n}^{b_{n}} \chi \mathrm{d} \mu & \leq \int \mathrm{T}_{n}^{g_{n}} \chi \cdot \mathbb{1}_{\Gamma_{n}} \mathrm{~d} \mu+\sum_{k=1}^{\infty} \int \mathrm{T}_{n}^{2^{k} \cdot g_{n}} \chi \cdot \mathbb{1}_{\Delta_{k, n}} \mathrm{~d} \mu \\
& \leq \int \mathrm{T}_{n}^{g_{n}} \chi \mathrm{d} \mu+\sum_{k=1}^{\infty} \int \mathrm{T}_{n}^{2^{k} \cdot g_{n}} \chi \cdot \mathbb{1}_{\Phi_{k, n}} \mathrm{~d} \mu .
\end{aligned}
$$

We will show in the following that $\sum_{k=1}^{\infty} \int \mathrm{T}_{n}^{2^{k}} \cdot g_{n} \chi \cdot \mathbb{1}_{\Phi_{k, n}} \mathrm{~d} \mu$ is negligible compared to $\int \mathrm{T}_{n}^{g_{n}} \chi \mathrm{d} \mu$. First we define $r:=\min \{n \in \mathbb{N}: \boldsymbol{\psi}(n)<1\}$ and

$$
\Phi_{k, n}^{\prime}:=\left\{\#\left\{j \leq n: \chi \circ T^{j-1}>2^{k-1} \cdot g_{n}\right\}>b_{n}-2 r+1\right\} .
$$

Since $\Phi_{k, n}=\left\{\#\left\{j \leq n: \chi \circ T^{j-1}>2^{k-1} \cdot g_{n}\right\}>b_{n}\right\}$, we have that $\Phi_{k, n} \subset \Phi_{k, n}^{\prime}$. We will show (B) by proving the following three statements:

(B1) We have for all $k \geq 1$ and $n$ sufficiently large uniformly in $k$ that

$$
\int \mathrm{T}_{n}^{2^{k} \cdot g_{n}} \chi \cdot \mathbb{1}_{\Phi_{k, n}} \mathrm{~d} \mu \leq \frac{(1+\boldsymbol{\psi}(r))^{2}}{1-\boldsymbol{\psi}(r)} \cdot \int \mathrm{T}_{n}^{2^{k} \cdot g_{n}} \chi \mathrm{d} \mu \cdot \mu\left(\Phi_{k, n}^{\prime}\right) .
$$

(B2) We have

$$
\sum_{k=2}^{\infty} \int \mathrm{T}_{n}^{2^{k} \cdot g_{n}} \chi \mathrm{d} \mu \cdot \mu\left(\Phi_{k, n}^{\prime}\right)=o\left(\int \mathrm{T}_{n}^{g_{n}} \chi \mathrm{d} \mu\right)
$$

(B3) We have

$$
\int \mathrm{T}_{n}^{2 \cdot g_{n}} \chi \mathrm{d} \mu \cdot \mu\left(\Phi_{1, n}^{\prime}\right)=o\left(\int \mathrm{T}_{n}^{g_{n}} \chi \mathrm{d} \mu\right)
$$


Combining these statements with (11) proves (B) In (B1) we have used a short notation which we will also use in the sequel. If we write that a statement $A_{k, n}$ depending on $n$ and $k$ holds for $n$ sufficiently large uniformly in $k$ we mean that there exists $N \in \mathbb{N}$ such that the statement $A_{n, k}$ holds for all $n \geq N$ and all $k$.

Proof of (B1) We will start this section with a set of definitions explaining in the sequel the strategy of the proof. Let

$$
E_{k, n, m, 1}:=\left\{\chi \circ T^{m-1} \leq 2^{k-1} \cdot g_{n}\right\} \text { and } E_{k, n, m, 2}:=\left\{\chi \circ T^{m-1}>2^{k-1} \cdot g_{n}\right\} \text { and } E_{k, n, m, 3}:=\Omega \text {. }
$$

Further, for $J \in\{1,2,3\}^{n}$ let $D_{k, n, J}:=\bigcap_{J=\left(j_{m}\right)_{1 \leq m \leq n}} E_{k, n, m, j_{m}}$. Loosely speaking our set $D_{k, n, J}$ determines for each $m \leq n$ if $\chi \circ T^{m-1}>2^{k-1} \cdot g_{n}$ or $\chi \circ T^{m-1} \leq 2^{k-1} \cdot g_{n}$ holds, or if no information about $\chi \circ T^{m-1}$ is gained.

Remember that $r=\min \{n \in \mathbb{N}: \boldsymbol{\psi}(n)<1\}$. Further, let $\Gamma_{n, i}:=\left\{m \in \mathbb{N}_{\leq n}:|m-i| \geq r\right\}$ and let

$$
\begin{gathered}
\mathcal{J}_{k, n}^{i}:=\left\{J=\left(j_{m}\right)_{1 \leq m \leq n} \in\{1,2,3\}^{n}: j_{m} \neq 3 \text { for } m \in \Gamma_{n, i} \text { and } j_{m}=3 \text { for } m \in\left(\Gamma_{n, i}\right)^{c}\right. \\
\text { and } \left.\#\left\{m \in \Gamma_{n, i}: \chi \circ T^{m-1}>2^{k-1} \cdot g_{n}\right\}>b_{n}-2 r+1\right\} .
\end{gathered}
$$

Here and in the following we denote by $A^{c}$ the complement of a set $A$. Since $\# \Gamma_{n, i}^{c} \leq 2 r-1$, the definition of $\mathcal{J}_{k, n}^{i}$ implies for each $k, n \in \mathbb{N}$ and $i \in \mathbb{N}_{\leq n}$ that

$$
\Phi_{k, n} \subset \biguplus_{J \in \mathcal{J}_{k, n}^{i}} D_{k, n, J} \subset \Phi_{k, n}^{\prime} .
$$

Thus, we have for each $i \in \mathbb{N}_{\leq n}$ and $k, n \in \mathbb{N}$

$$
\int \mathrm{T}_{n}^{2^{k} \cdot g_{n}} \chi \cdot \mathbb{1}_{\Phi_{k, n}} \mathrm{~d} \mu=\sum_{i=1}^{n} \int 2^{2^{k} \cdot g_{n}} \chi \circ T^{i-1} \cdot \mathbb{1}_{\Phi_{k, n}} \mathrm{~d} \mu \leq \sum_{i=1}^{n} \sum_{J \in \mathcal{J}_{k, n}^{i}} \int 2^{2^{k} \cdot g_{n}} \chi \circ T^{i-1} \cdot \mathbb{1}_{D_{k, n, J}} \mathrm{~d} \mu .
$$

For each $k, n \in \mathbb{N}, i \in \mathbb{N}_{\leq n}$, and $J \in \mathcal{J}_{k, n}^{i}$ we can write $D_{k, n, J}$ as an intersection of two events $D_{k, n, J}^{i,<} \cap D_{k, n, J}^{i,>}$, where

$$
D_{k, n, J}^{i,<}:=\bigcap_{\left(j_{m}\right)=J, m=1, \ldots, i-r} E_{k, n, m, j_{m}} \quad \text { and } \quad D_{k, n, J}^{i,>}:=\bigcap_{\left(j_{m}\right)=J, m=i+r, \ldots, n} E_{k, n, m, j_{m}} .
$$

If $i-r<1$, then we set $D_{k, n, J}^{i,<}:=\Omega$ and if $i+r>n$, then we set $D_{k, n, J}^{i,>}:=\Omega$. Loosely speaking, $D_{k, n, J}^{i,<}$ is determined by the information of the first $i-r$ entries and $D_{k, n, J}^{i,>}$ of the last entries starting from the $(i+r)$-th. We notice that for $J \in \mathcal{J}_{n, k}^{i}$

$$
\int 2^{2^{k} \cdot g_{n}} \chi \circ T^{i-1} \cdot \mathbb{1}_{D_{k, n, J}} \mathrm{~d} \mu=\int \mathbb{1}_{D_{k, n, J}^{i,<}} \cdot 2^{2^{k} \cdot g_{n}} \chi \circ T^{i-1} \cdot \mathbb{1}_{D_{k, n, J}^{i,>}} \mathrm{d} \mu .
$$

To estimate this term we will use the $r$ th $\boldsymbol{\psi}$-mixing coefficient for which by assumption we have that $\boldsymbol{\psi}(r)<1$. To make use of the $\boldsymbol{\psi}$-mixing property we notice that for any random variables $X, Y$

$$
\operatorname{Cov}(X, Y) \leq \boldsymbol{\psi}(\sigma(X), \sigma(Y)) \cdot|X|_{1} \cdot|Y|_{1},
$$

where we denote by Cov the covariance, see e.g. [Dou94, Theorem 3, Chapter 1.2.2]. This implies for non-negative random variables

$$
\int X \cdot Y \mathrm{~d} \mu \leq(1+\boldsymbol{\psi}(\sigma(X), \sigma(Y))) \cdot \int X \mathrm{~d} \mu \cdot \int Y \mathrm{~d} \mu .
$$


The following statements will all hold for all $i \in \mathbb{N}_{\leq N}, k, n \in \mathbb{N}$ and $J \in \mathcal{J}_{k, n}^{i}$. For brevity we will not mention that for each of the following calculations. By noticing that

$$
\begin{gathered}
\boldsymbol{\psi}\left(\sigma\left(\mathbb{1}_{D_{k, n, J}^{i,<}}\right), \sigma\left(2^{k \cdot g_{n}} \chi \circ T^{i-1} \cdot \mathbb{1}_{D_{k, n, J}^{i,>}}\right)\right) \leq \boldsymbol{\psi}(r) \text { and } \\
\boldsymbol{\psi}\left(\sigma\left(2^{k \cdot g_{n}} \chi \circ T^{i-1}\right), \sigma\left(\mathbb{1}_{D_{k, n, J}^{i,>}}\right)\right) \leq \boldsymbol{\psi}(r)
\end{gathered}
$$

we obtain

$$
\begin{aligned}
& \int 2^{k} \cdot g_{n} \chi \circ T^{i-1} \cdot \mathbb{1}_{D_{k, n, J}} \mathrm{~d} \mu \\
& =\int \mathbb{1}_{D_{k, n, J}^{i,<}} \cdot 2^{k} \cdot g_{n} \chi \circ T^{i-1} \cdot \mathbb{1}_{D_{k, n, J}^{i,>}} \mathrm{d} \mu \\
& \leq(1+\psi(r)) \int \mathbb{1}_{D_{k, n, J}^{i,<}} \mathrm{d} \mu \cdot \int 2^{k} \cdot g_{n} \chi \circ T^{i-1} \cdot \mathbb{1}_{D_{k, n, J}^{i,>}} \mathrm{d} \mu \\
& \leq(1+\boldsymbol{\psi}(r)) \int \mathbb{1}_{D_{k, n, J}^{i,<}} \mathrm{d} \mu \cdot(1+\boldsymbol{\psi}(r)) \int 2^{2^{k} \cdot g_{n}} \chi \circ T^{i-1} \mathrm{~d} \mu \cdot \int \mathbb{1}_{D_{k, n, J}^{i,>}} \mathrm{d} \mu \\
& =(1+\boldsymbol{\psi}(r))^{2} \cdot \mu\left(D_{k, n, J}^{i,<}\right) \cdot \mu\left(D_{k, n, J}^{i,>}\right) \cdot \int 2^{k} \cdot g_{n} \chi \circ T^{i-1} \mathrm{~d} \mu .
\end{aligned}
$$

Using (14) in the other direction gives for non-negative random variables

$$
\int X \mathrm{~d} \mu \cdot \int X \mathrm{~d} \mu \leq \frac{\int X \cdot Y \mathrm{~d} \mu}{1-\psi(\sigma(X), \sigma(Y))}
$$

yielding

$$
\mu\left(D_{k, n, J}^{i,<}\right) \cdot \mu\left(D_{k, n, J}^{i,>}\right) \leq \frac{\mu\left(D_{k, n, J}\right)}{1-\boldsymbol{\psi}(r)}
$$

and thus

$$
\int 2^{2^{k} \cdot g_{n}} \chi \circ T^{i-1} \cdot \mathbb{1}_{D_{k, n, J}} \mathrm{~d} \mu \leq \frac{(1+\boldsymbol{\psi}(r))^{2}}{1-\boldsymbol{\psi}(r)} \cdot \mu\left(D_{k, n, J}\right) \cdot \int{ }^{2^{k} \cdot g_{n}} \chi \circ T^{i-1} \mathrm{~d} \mu .
$$

Combining this with (12) and (13) implies (B1)

Proof of (B2), We will first estimate $\int \mathrm{T}_{n}^{2^{k} \cdot g_{n}} \chi \mathrm{d} \mu$ using (7) and applying Potter's bound, see e.g. [BGT87, Theorem 1.5.6], which gives

$$
\begin{aligned}
\int \mathrm{T}_{n}^{2^{k} \cdot g_{n}} \chi \mathrm{d} \mu & \leq n \cdot 2 \cdot \frac{\alpha}{1-\alpha} \cdot L\left(2^{k} \cdot g_{n}\right) \cdot\left(2^{k} \cdot g_{n}\right)^{1-\alpha} \\
& \leq n \cdot 2^{k+1} \cdot \frac{\alpha}{1-\alpha} \cdot L\left(g_{n}\right) \cdot g_{n}^{1-\alpha} \\
& \leq 2^{k+1} \cdot \int \mathrm{T}_{n}^{g_{n}} \chi \mathrm{d} \mu,
\end{aligned}
$$

for $n$ sufficiently large uniformly in $k$.

Next, we will estimate $\mu\left(\Phi_{k, n}^{\prime}\right)$. We will use two different techniques, one for rather small and the other for larger $k$. We start with the estimate for the smaller $k$. To ease notation we set $\overline{\mathbb{1}}_{A}:=\mathbb{1}_{A}-\mu(A)$, for any measurable set $A$. We notice that for all $k, n \in \mathbb{N}$,

$$
\begin{aligned}
\mu\left(\Phi_{k, n}^{\prime}\right) & =\mu\left(\sum_{i=1}^{n} \mathbb{1}_{\left\{\chi \circ T^{i-1}>2^{k-1} \cdot g_{n}\right\}}>b_{n}-2 r+1\right) \\
& =\mu\left(\sum_{i=1}^{n} \overline{\mathbb{1}}_{\left\{\chi \circ T^{i-1}>2^{k-1} \cdot g_{n}\right\}}>b_{n}-2 r+1-n \cdot \mu\left(\chi>2^{k-1} \cdot g_{n}\right)\right) \\
& \leq \mu\left(\left|\sum_{i=1}^{n} \overline{\mathbb{1}}_{\left\{\chi \circ T^{i-1}>2^{k-1} \cdot g_{n}\right\}}\right|>b_{n}-2 r+1-n \cdot \mu\left(\chi>2^{k-1} \cdot g_{n}\right)\right) .
\end{aligned}
$$


Furthermore, applying the definition of the distribution function and Potter's bound implies

$$
n \cdot \mu\left(\chi>2^{k-1} \cdot g_{n}\right)=n \cdot \frac{L\left(2^{k-1} \cdot g_{n}\right)}{\left(2^{k-1} \cdot g_{n}\right)^{\alpha}} \leq n \cdot \frac{L\left(g_{n}\right)}{2^{(k-1) \cdot \alpha / 2} \cdot g_{n}^{\alpha}}=\frac{n \cdot\left(1-F\left(g_{n}\right)\right)}{2^{(k-1) \cdot \alpha / 2}},
$$

for $n$ sufficiently large uniformly in $k \geq 2$. Next, we aim to prove that $\left(1-F\left(g_{n}\right)\right) \sim b_{n} / n$. On the one hand we have that $F\left(F^{\leftarrow}(x)\right) \geq x$ and on the other hand $F\left(F^{\leftarrow}(x)-1\right) \leq x$. Hence, the definition of $\left(g_{n}\right)$ in (8) gives

$$
\begin{aligned}
\frac{b_{n}}{n} & \sim \frac{b_{n}-\zeta_{n}}{n} \geq 1-F\left(F^{\leftarrow}\left(1-\frac{b_{n}-\zeta_{n}}{n}\right)\right)=1-F\left(g_{n}\right)=\frac{L\left(g_{n}\right)}{\left(g_{n}\right)^{\alpha}} \sim \frac{L\left(g_{n}-1\right)}{\left(g_{n}-1\right)^{\alpha}} \\
& =1-F\left(g_{n}-1\right)=1-F\left(F^{\leftarrow}\left(1-\frac{b_{n}-\zeta_{n}}{n}\right)-1\right) \geq \frac{b_{n}-\zeta_{n}}{n} \sim \frac{b_{n}}{n}
\end{aligned}
$$

and thus

$$
n \cdot \mu\left(\chi>2^{k-1} \cdot g_{n}\right)<\frac{b_{n} \cdot 2^{3 / 4 \cdot \alpha}}{2^{k \cdot \alpha / 2}},
$$

for $n$ sufficiently large uniformly in $k \geq 2$ and thus

$$
b_{n}-2 r+1-n \cdot \mu\left(\chi>2^{k-1} \cdot g_{n}\right) \geq \frac{2^{\alpha / 4}-1}{2^{\alpha / 4}} \cdot b_{n}-2 r+1 \geq \frac{2^{\alpha / 4}-1}{2^{\alpha}} \cdot b_{n},
$$

for $n$ sufficiently large uniformly in $k \geq 2$.

In order to proceed we will make use of the following lemma.

Lemma 2.3 (KS18, Lemma 2.9]). Let $(\Omega, \mathcal{A}, T, \mu, \mathcal{F},\|\cdot\|)$ fulfill Property $\mathfrak{C}$. Then there exist positive constants $K, N, U$ such that for all $\varphi \in \mathcal{F}$ fulfilling $\int \varphi \mathrm{d} \mu=0$, all $u \in \mathbb{R}_{>0}$, and all $n \in \mathbb{N}_{>N}$ we have

$$
\mu\left(\max _{i \leq n}\left|S_{i} \varphi\right| \geq u\right) \leq K \cdot \exp \left(-U \cdot \frac{u}{\|\varphi\|} \cdot \min \left\{\frac{u}{n \cdot|\varphi|_{1}}, 1\right\}\right) .
$$

Combining (18), (22), and Lemma 2.3 yields

$$
\begin{aligned}
& \mu\left(\Phi_{k, n}^{\prime}\right) \leq \mu\left(\left|\sum_{i=1}^{n} \overline{\mathbb{1}}_{\left\{\chi \circ T^{i-1}>2^{k-1} \cdot g_{n}\right\}}\right|>\frac{2^{\alpha / 4}-1}{2^{\alpha}} \cdot b_{n}\right) \\
& \leq K \cdot \exp \left(-U \cdot \frac{2^{\alpha / 4}-1}{2^{\alpha}} \cdot \frac{b_{n}}{\left\|\overline{\mathbb{1}}_{\left\{\chi>2^{k-1} \cdot g_{n}\right\}}\right\|}\right. \\
&\left.\cdot \min \left\{\frac{2^{\alpha / 4}-1}{2^{\alpha}} \cdot \frac{b_{n}}{n \cdot\left|\overline{\mathbb{1}}_{\left\{\chi>2^{k-1} \cdot g_{n}\right\}}\right|_{1}}, 1\right\}\right),
\end{aligned}
$$

for $n$ sufficiently large uniformly in $k \geq 2$. Furthermore, we have that

$$
\left|\overline{\mathbb{1}}_{\left\{\chi>2^{k-1} \cdot g_{n}\right\}}\right|_{1}=2 \cdot \frac{L\left(2^{k-1} \cdot g_{n}\right)}{\left(2^{k-1} \cdot g_{n}\right)^{\alpha}} \cdot\left(1-\frac{L\left(2^{k-1} \cdot g_{n}\right)}{\left(2^{k-1} \cdot g_{n}\right)^{\alpha}}\right) \leq 2 \cdot \frac{L\left(2^{k-1} \cdot g_{n}\right)}{\left(2^{k-1} \cdot g_{n}\right)^{\alpha}}
$$

and (21) implies

$$
\left|\overline{\mathbb{1}}_{\left\{\chi>2^{k-1} \cdot g_{n}\right\}}\right|_{1} \leq 4 \cdot \frac{b_{n}}{2^{\alpha / 2 \cdot k} \cdot n},
$$

for $n$ sufficiently large uniformly in $k \geq 2$. With $K_{1}$ defined in (4) we get

$$
\begin{aligned}
\left\|\overline{\mathbb{1}}_{\left\{\chi>2^{k-1} \cdot g_{n}\right\}}\right\| & \leq\left\|\mathbb{1}_{\left\{\chi>2^{k-1} \cdot g_{n}\right\}}\right\|+\left\|\mu\left(\chi>2^{k-1} \cdot g_{n}\right)\right\| \\
& =\left\|\mathbb{1}_{\left\{\chi>2^{k-1} \cdot g_{n}\right\}}\right\|+\mu\left(\chi>2^{k-1} \cdot g_{n}\right)\|\mathbb{1}\| \leq K_{1}+\|\mathbb{1}\|
\end{aligned}
$$


and thus an application of (23), (24), and (25) gives

$$
\begin{aligned}
\mu\left(\Phi_{k, n}^{\prime}\right) & \leq K \cdot \exp \left(-U \cdot \frac{2^{\alpha / 2}-1}{2^{\alpha}} \cdot \frac{b_{n}}{K_{1}+\|1\|} \cdot \min \left\{\frac{\left(2^{\alpha / 2}-1\right) \cdot 2^{\alpha / 2 \cdot k}}{4}, 1\right\}\right) \\
& =K \cdot \exp \left(-W_{\alpha} \cdot b_{n}\right),
\end{aligned}
$$

for $n$ sufficiently large uniformly in $k \geq 2$, where

$$
W_{\alpha}:=U \cdot \frac{\left(2^{\alpha / 2}-1\right)^{2}}{2^{\alpha+2} \cdot\left(K_{1}+\|1\|\right)} .
$$

By using this estimate and (17) we can further estimate

$$
\int \mathrm{T}_{n}^{2^{k} \cdot g_{n}} \chi \mathrm{d} \mu \cdot \mu\left(\Phi_{k, n}^{\prime}\right) \leq K \cdot 2^{k+1} \cdot \exp \left(-W_{\alpha} \cdot b_{n}\right) \cdot \int \mathrm{T}_{n}^{g_{n}} \chi \mathrm{d} \mu .
$$

This will later give us the estimate for small $k$, let us proceed with an estimate for large values of $k$. Let $m:=\lfloor 2 / \alpha\rfloor+1$. There exists $N \in \mathbb{N}$ such that for all $n \geq N$ and $k \in \mathbb{N}$ we have

$$
\Phi_{k, n}^{\prime} \subset\left\{\#\left\{j \leq n: \chi \circ T^{j-1}>2^{k-1} \cdot g_{n}\right\} \geq m \cdot r\right\}=: \Upsilon_{k, n}
$$

We have that

$$
\Upsilon_{k, n} \subset \underset{\substack{1 \leq i_{1}<\ldots<i_{m} \leq n \\ i_{j}-i_{j-1} \geq r, j=2, \ldots, n}}{\bigcup} \bigcap_{j=1}^{m}\left\{\chi \circ T^{i_{j}-1}>2^{k-1} \cdot g_{n}\right\}
$$

and thus

$$
\mu\left(\Upsilon_{k, n}\right) \leq \sum_{\substack{1 \leq i_{1}<\ldots<i_{m} \leq n \\ i_{j}-i_{j-1} \geq r, j=2, \ldots, n}} \mu\left(\bigcap_{j=1}^{m}\left\{\chi \circ T^{i_{j}-1}>2^{k-1} \cdot g_{n}\right\}\right)
$$

Each of the summands can be estimated using (15):

$$
\begin{aligned}
\mu\left(\bigcap_{j=1}^{m}\left\{\chi \circ T^{i_{j}-1}>2^{k-1} \cdot g_{n}\right\}\right) \\
\quad \leq \mu\left(\chi \circ T^{i_{1}-1}>2^{k-1} \cdot g_{n}\right) \cdot \mu\left(\bigcap_{j=2}^{m}\left\{\chi \circ T^{i_{j}-1}>2^{k-1} \cdot g_{n}\right\}\right) \cdot\left(1+\boldsymbol{\psi}\left(i_{2}-i_{1}\right)\right) \\
\quad \leq \mu\left(\chi \circ T^{i_{1}-1}>2^{k-1} \cdot g_{n}\right) \cdot \mu\left(\bigcap_{j=2}^{m}\left\{\chi \circ T^{i_{j}-1}>2^{k-1} \cdot g_{n}\right\}\right) \cdot(1+\boldsymbol{\psi}(r)) \\
\quad \vdots \\
\quad \leq \prod_{j=1}^{m} \mu\left(\chi \circ T^{i_{j}-1}>2^{k-1} \cdot g_{n}\right) \cdot(1+\boldsymbol{\psi}(r))^{m-1} \\
\quad=\mu\left(\chi>2^{k-1} \cdot g_{n}\right)^{m} \cdot(1+\boldsymbol{\psi}(r))^{m-1} \\
\quad \leq \mu\left(\chi>2^{k-1} \cdot g_{n}\right)^{m} \cdot 2^{m-1} .
\end{aligned}
$$

We have that

$$
\#\left\{\left(i_{1}, \ldots, i_{m}\right): 1 \leq i_{1}<\ldots<i_{m} \leq n\right\}=\left(\begin{array}{c}
n \\
m
\end{array}\right)<n^{m}
$$

implying

$$
\mu\left(\Upsilon_{k, n}\right) \leq\left(n \cdot \mu\left(\chi>2^{k-1} \cdot g_{n}\right)\right)^{m} \cdot 2^{m-1}
$$


With (21) we conclude

$$
\mu\left(\Upsilon_{k, n}\right) \leq\left(\frac{2 \cdot b_{n}}{2^{k \cdot \alpha / 2}}\right)^{m} \cdot 2^{m-1}
$$

for $n$ sufficiently large uniformly in $k \geq 2$. Combining this estimate with (17) and (28) gives

$$
\int \mathrm{T}_{n}^{2^{k} \cdot g_{n}} \chi \mathrm{d} \mu \cdot \mu\left(\Phi_{k, n}^{\prime}\right) \leq 2^{2 m} \cdot 2^{k \cdot(1-\alpha / 2 \cdot m)} \cdot b_{n}^{m} \cdot \int \mathrm{T}_{n}^{g_{n}} \chi \mathrm{d} \mu
$$

for $n$ sufficiently large uniformly in $k \geq 2$.

Finally, we combine the estimates for small and large $k$ given in (27) and (29) to obtain

$$
\begin{aligned}
& \sum_{k=2}^{\infty} \int \mathrm{T}_{n}^{2^{k} \cdot g_{n}} \chi \mathrm{d} \mu \cdot \mu\left(\Phi_{k, n}^{\prime}\right) \\
& \quad \leq\left(\sum_{k=2}^{b_{n}^{1 / 2}-1} K \cdot 2^{k+1} \cdot \exp \left(-W_{\alpha} \cdot b_{n}\right)+\sum_{k=b_{n}^{1 / 2}}^{\infty} 2^{2 m} \cdot 2^{k \cdot(1-\alpha / 2 \cdot m)} \cdot b_{n}^{m}\right) \cdot \int \mathrm{T}_{n}^{g_{n}} \chi \mathrm{d} \mu .
\end{aligned}
$$

Estimating the sums of the first factor of (30) separately implies

$$
\begin{aligned}
\sum_{k=2}^{b_{n}^{1 / 2}-1} K \cdot 2^{k+1} \cdot \exp \left(-W_{\alpha} \cdot b_{n}\right) & <K \cdot 2^{b_{n}^{1 / 2}+1} \cdot \exp \left(-W_{\alpha} \cdot b_{n}\right) \\
& =K \cdot \exp \left(\log 2 \cdot\left(b_{n}^{1 / 2}+1\right)-W_{\alpha} \cdot b_{n}\right) \\
& \leq K \cdot \exp \left(-W_{\alpha} / 2 \cdot b_{n}\right),
\end{aligned}
$$

for $n$ sufficiently large and

$$
\sum_{k=b_{n}^{1 / 2}}^{\infty} 2^{2 m} \cdot 2^{k \cdot(1-\alpha / 2 \cdot m)} \cdot b_{n}^{m} \leq 2^{2 m} \cdot \frac{2^{b_{n}^{1 / 2} \cdot(1-\alpha / 2 \cdot m)}}{1-2^{1-\alpha / 2 \cdot m}} \cdot b_{n}^{m}
$$

for $n$ sufficiently large. Since we chose $m>2 / \alpha$, (32) tends to zero for $n$ tending to infinity. Combining (31) and (32) with (30) proves the statement of (B2).

Proof of (B3) In order to consider the case $k=1$ we notice that by the definition of $\left(g_{n}\right)$ in (8) and the fact that $F\left(F^{\leftarrow}(x)\right) \leq x$ we have that

$$
\mu\left(\chi>g_{n}\right)=1-F\left(g_{n}\right)=1-F\left(F^{\leftarrow}\left(1-\frac{b_{n}-\zeta_{n}}{n}\right)\right) \leq \frac{b_{n}-\zeta_{n}}{n}
$$

and thus $b_{n}-n \cdot \mu\left(\chi>g_{n}\right) \geq \zeta_{n}$. If we combine this with (18), we can conclude

$$
\mu\left(\Phi_{1, n}^{\prime}\right) \leq \mu\left(\left|\sum_{i=1}^{n} \overline{\mathbb{1}}_{\left\{\chi \circ T^{i-1}>g_{n}\right\}}\right| \geq \zeta_{n}-2 r+1\right) \leq \mu\left(\left|\sum_{i=1}^{n} \overline{\mathbb{1}}_{\left\{\chi \circ T^{i-1}>g_{n}\right\}}\right| \geq \zeta_{n} / 2\right)
$$

for $n$ sufficiently large. Using Lemma 2.3 implies

$$
\mu\left(\Phi_{1, n}^{\prime}\right) \leq K \cdot \exp \left(-\frac{U \cdot \zeta_{n}}{2 \cdot\left\|\overline{\mathbb{1}}_{\left\{\chi>g_{n}\right\}}\right\|} \cdot \min \left\{\frac{\zeta_{n}}{2 \cdot n \cdot\left|\overline{\mathbb{1}}_{\left\{\chi>g_{n}\right\}}\right|_{1}}, 1\right\}\right)
$$

for $n$ sufficiently large. Using (20), we have that

$$
\left|\overline{\mathbb{1}}_{\left\{\chi>g_{n}\right\}}\right|_{1} \leq 2 \mu\left(\chi>g_{n}\right)=2 \cdot\left(1-F\left(g_{n}\right)\right) \leq 3 b_{n} / n,
$$

for $n$ sufficiently large. Therefore,

$$
\min \left\{\frac{\zeta_{n}}{2 \cdot n \cdot\left|\overline{\mathbb{1}}_{\left\{\chi>g_{n}\right\}}\right|_{1}}, 1\right\} \geq \min \left\{\frac{\zeta_{n}}{n \cdot 6 b_{n} / n}, 1\right\}=\min \left\{\frac{\zeta_{n}}{6 b_{n}}, 1\right\}=\frac{\zeta_{n}}{6 b_{n}},
$$


for $n$ sufficiently large. Combining (25) with (34) and (35) and using the definition of $\left(\zeta_{n}\right)$ yields for $n$ sufficiently large that

$$
\mu\left(\Phi_{1, n}^{\prime}\right) \leq K \cdot \exp \left(-\frac{U \cdot \zeta_{n}}{2\left(K_{1}+\|\mathbb{1}\|\right)} \cdot \frac{\zeta_{n}}{6 b_{n}}\right)=K \cdot \exp \left(-\frac{U \cdot b_{n}^{1 / 3}}{12\left(K_{1}+\|\mathbb{1}\|\right)}\right),
$$

for $n$ sufficiently large which tends to zero for $n$ tending to infinity. If we combine this with (17), we obtain (B3)

Proof of $(C)$. We set $\bar{\Phi}_{n}:=\left\{\#\left\{i \leq n: \chi \circ T^{i-1}>g_{n}\right\}<n \cdot \mu\left(\chi>g_{n}\right)-\zeta_{n}\right\}$ and have that

$$
\begin{aligned}
\int \mathrm{S}_{n}^{b_{n}} \chi \mathrm{d} \mu & \geq \int \mathrm{S}_{n}^{b_{n}} \chi \cdot \mathbb{1}_{\bar{\Phi}_{n}^{c}} \mathrm{~d} \mu \geq \int \mathrm{T}_{n}^{g_{n}} \chi \cdot \mathbb{1}_{\bar{\Phi}_{n}^{c}} \mathrm{~d} \mu-\left(b_{n}-n \cdot \mu\left(\chi>g_{n}\right)+\zeta_{n}\right) \cdot g_{n} \\
& =\int \mathrm{T}_{n}^{g_{n}} \chi \mathrm{d} \mu-\int \mathrm{T}_{n}^{g_{n}} \chi \cdot \mathbb{1}_{\bar{\Phi}_{n}} \mathrm{~d} \mu-\left(b_{n}-n \cdot \mu\left(\chi>g_{n}\right)+\zeta_{n}\right) \cdot g_{n} .
\end{aligned}
$$

It is sufficient to show

$$
\int \mathrm{T}_{n}^{g_{n}} \chi \cdot \mathbb{1}_{\bar{\Phi}_{n}} \mathrm{~d} \mu=o\left(\int \mathrm{T}_{n}^{g_{n}} \chi \mathrm{d} \mu\right)
$$

and

$$
\left(b_{n}-n \cdot \mu\left(\chi>g_{n}\right)+\zeta_{n}\right) \cdot g_{n}=o\left(\int \mathrm{T}_{n}^{g_{n}} \chi \mathrm{d} \mu\right) .
$$

We start with showing (38). We have by (7) and the definition of $F$ that

$$
\int \mathrm{T}_{n}^{g_{n}} \chi \mathrm{d} \mu \sim \frac{\alpha}{1-\alpha} \cdot n \cdot g_{n}^{1-\alpha} \cdot L\left(g_{n}\right)=\frac{\alpha}{1-\alpha} \cdot n \cdot\left(1-F\left(g_{n}\right)\right) \cdot g_{n} .
$$

This together with (20) shows (38).

Let us look at the asymptotic (37). Similarly as in the proof of (B1) set

$$
\begin{gathered}
\mathcal{K}_{n}^{i}:=\left\{J=\left(j_{m}\right)_{1 \leq m \leq n} \in\{1,2,3\}^{n}: j_{m} \neq 3 \text { for } m \in \Gamma_{n, i} \text { and } j_{m}=3 \text { for } m \in\left(\Gamma_{n, i}\right)^{c}\right. \\
\text { and \# } \left.\left\{m \in \Gamma_{n, i}: \chi \circ T^{m-1}>g_{n}\right\}<b_{n}-2 \zeta_{n}+2 r-1\right\} .
\end{gathered}
$$

This implies

$$
\bar{\Phi}_{n} \subset \biguplus_{J \in \mathcal{K}_{n}^{i}} D_{k, n, J} \subset\left\{\#\left\{m \leq n: \chi \circ T^{m-1}>g_{n}\right\}<n \cdot \mu\left(\chi>g_{n}\right)-\zeta_{n}+2 r-1\right\}=: \bar{\Phi}_{n}^{\prime} .
$$

Using an analogous argument as in (B1) we obtain with the help of (16) that

$$
\begin{aligned}
\int \mathrm{T}_{n}^{g_{n}} \chi \cdot \mathbb{1}_{\bar{\Phi}_{n}} \mathrm{~d} \mu & \leq \sum_{i=1}^{n} \sum_{J \in \mathcal{K}_{n}^{i}}{ }^{g_{n}} \chi \circ T^{i-1} \cdot \mathbb{1}_{D_{k, n, J}} \mathrm{~d} \mu \\
& \leq \sum_{i=1}^{n} \sum_{J \in \mathcal{K}_{n}^{i}} \frac{(1+\psi(r))^{2}}{1-\psi(r)} \cdot \int{ }^{g_{n}} \chi \circ T^{i-1} \mathrm{~d} \mu \cdot \mu\left(D_{k, n, J}\right) \\
& \leq \frac{(1+\boldsymbol{\psi}(r))^{2}}{1-\boldsymbol{\psi}(r)} \cdot \int \mathrm{T}_{n}^{g_{n}} \chi \mathrm{d} \mu \cdot \mu\left(\bar{\Phi}_{n}^{\prime}\right) .
\end{aligned}
$$

In the next steps we estimate $\mu\left(\bar{\Phi}_{n}^{\prime}\right)$. We have that

$$
\mu\left(\bar{\Phi}_{n}^{\prime}\right)=\mu\left(\sum_{i=1}^{n} \overline{\mathbb{1}}_{\left\{\chi \circ T^{i-1}>g_{n}\right\}}<-\zeta_{n}+2 r-1\right) \leq \mu\left(\left|\sum_{i=1}^{n} \overline{\mathbb{1}}_{\left\{\chi \circ T^{i-1}>g_{n}\right\}}\right|>\zeta_{n} / 2\right),
$$

for $n$ sufficiently large. Hence, (33) implies that we can estimate $\mu\left(\bar{\Phi}_{n}^{\prime}\right)$ in the same manner as $\mu\left(\Phi_{1, n}^{\prime}\right)$ and obtain by (36) that we have for sufficiently large $n$ that $\mu\left(\bar{\Phi}_{n}^{\prime}\right) \leq \exp \left(U \cdot b_{n}^{1 / 3} /\left(12\left(K_{1}+\right.\right.\right.$ $\|\mathbb{1}\|))$ ) which tends to zero for $n$ tending to infinity. Combining this observation with (39) proves (37) which was the final step in the proof of (C). 
Proof of Lemma 2.2. We make use of the following upper estimate:

$$
\mu\left(\left|\frac{S_{n}^{b_{n}} \chi}{d_{n}}-1\right|>\epsilon\right) \leq \mu\left(\frac{S_{n}^{b_{n}} \chi}{d_{n}}-1>\epsilon\right)+\mu\left(\frac{S_{n}^{b_{n}} \chi}{d_{n}}-1<-\epsilon\right)
$$

and we will show that both terms on the right-hand side tend to zero for $n$ tending to infinity. In order to estimate the first summand of (40) we note that

$$
\mu\left(\frac{\mathrm{S}_{n}^{b_{n}} \chi}{d_{n}}-1>\epsilon\right) \leq \mu\left(\mathrm{T}_{n}^{g_{n}} \chi<\mathrm{S}_{n}^{b_{n}} \chi\right)+\mu\left(\frac{\mathrm{T}_{n}^{g_{n}} \chi}{d_{n}}-1>\epsilon\right) .
$$

With $\Phi_{k, n}$ given in (10) we also have $\left\{\mathrm{T}_{n}^{g_{n}} \chi<\mathrm{S}_{n}^{b_{n}} \chi\right\}=\Phi_{1, n}$. An application of (12) and (26) implies that $\mu\left(\Phi_{1, n}\right)$ tends to zero for $n$ tending to infinity.

In order to estimate $\mu\left(\mathrm{T}_{n}^{g_{n}} \chi / d_{n}-1>\epsilon\right)$ we note that (9) implies $d_{n} \sim \int \mathrm{T}_{n}^{g_{n}} \chi \mathrm{d} \mu$. If we set $\overline{\mathrm{T}}_{n}^{r} \chi=\mathrm{T}_{n}^{r} \chi-\int \mathrm{T}_{n}^{r} \chi \mathrm{d} \mu$, then

$$
\mu\left(\frac{\mathrm{T}_{n}^{g_{n}} \chi}{d_{n}}-1>\epsilon\right) \leq \mu\left(\overline{\mathrm{T}}_{n}^{g_{n}} \chi>\frac{\epsilon}{2} \cdot d_{n}\right)
$$

for $n$ sufficiently large, and an application of Lemma 2.3 hence gives

$$
\mu\left(\frac{\mathrm{T}_{n}^{g_{n}} \chi}{d_{n}}-1>\epsilon\right) \leq K \cdot \exp \left(-U \cdot \frac{\epsilon \cdot d_{n}}{2 \cdot\left\|^{g_{n}} \bar{\chi}\right\|} \cdot \min \left\{\frac{\epsilon \cdot d_{n}}{\left.\left.2 \cdot n \cdot\right|^{g_{n}} \bar{\chi}\right|_{1}}, 1\right\}\right)
$$

for $n$ sufficiently large where ${ }^{r} \bar{\chi}:={ }^{r} \chi-\int{ }^{r} \chi \mathrm{d} \mu$. We note that the first part of (44) implies

$$
\left\|^{g_{n}} \bar{\chi}\right\| \leq\left\|^{g_{n}} \chi\right\|+\left\|\int{ }^{g_{n}} \chi \mathrm{d} \mu\right\| \leq g_{n} \cdot K_{1}+\int{ }^{g_{n}} \chi \mathrm{d} \mu \cdot\|\mathbb{1}\| \leq g_{n} \cdot\left(K_{1}+\|\mathbb{1}\|\right)
$$

and $\left.\left.n \cdot\right|^{g_{n}} \bar{\chi}\right|_{1} \leq 2 n \cdot \int{ }^{g_{n}} \chi \mathrm{d} \mu=2 \int \mathrm{T}_{n}^{g_{n}} \chi \mathrm{d} \mu \leq 3 d_{n}$, for $n$ sufficiently large, where the last inequality follows from (91). Combining this with (41) and (42) yields

$$
\mu\left(\frac{\mathrm{T}_{n}^{g_{n}} \chi}{d_{n}}-1>\epsilon\right) \leq K \cdot \exp \left(-\frac{U \cdot \epsilon^{2}}{12 \cdot\left(K_{1}+\|\mathbb{1}\|\right)} \cdot \frac{d_{n}}{g_{n}}\right),
$$

for $n$ sufficiently large and $\epsilon$ sufficiently small. We obtain with an analogous calculation as in [KS18, Proof of Theorem 1.7] that

$$
\begin{aligned}
g_{n} & =F^{\leftarrow}\left(1-\frac{b_{n}-\zeta_{n}}{n}\right) \sim\left(\frac{n}{b_{n}-\zeta_{n}}\right)^{1 / \alpha} \cdot\left(L^{1 / \alpha}\right)^{\#}\left(\left(\frac{n}{b_{n}-\zeta_{n}}\right)^{1 / \alpha}\right) \\
& \sim\left(\frac{n}{b_{n}}\right)^{1 / \alpha} \cdot\left(L^{1 / \alpha}\right)^{\#}\left(\left(\frac{n}{b_{n}}\right)^{1 / \alpha}\right) .
\end{aligned}
$$

Hence, by the definition of $\left(d_{n}\right)$ in (5) we have that $d_{n} / g_{n} \sim \alpha /(1-\alpha) \cdot b_{n}$ and

$$
\mu\left(\frac{\mathrm{T}_{n}^{g_{n}} \chi}{d_{n}}-1>\epsilon\right) \leq K \cdot \exp \left(-\frac{U \cdot \epsilon^{2} \cdot(1-\alpha)}{13 \cdot\left(K_{1}+\|\mathbb{1}\|\right) \cdot \alpha} \cdot b_{n}\right)
$$

for $n$ sufficiently large. Consequently, the right-hand side tends to zero as $\left(b_{n}\right)$ tends to infinity.

Next we will estimate the second summand of (40). For arbitrary $\delta>0$ we have that

$$
\mu\left(\frac{\mathrm{S}_{n}^{b_{n}} \chi}{d_{n}}-1<-\epsilon\right) \leq \mu\left(\mathrm{T}_{n}^{(1-\delta) \cdot g_{n}} \chi>\mathrm{S}_{n}^{b_{n}} \chi\right)+\mu\left(\frac{\mathrm{T}_{n}^{(1-\delta) \cdot g_{n}} \chi}{d_{n}}-1<-\epsilon\right) .
$$

We first estimate

$$
\begin{aligned}
\mu\left(\mathrm{T}_{n}^{(1-\delta) \cdot g_{n}} \chi>\mathrm{S}_{n}^{b_{n}} \chi\right) & =\mu\left(\sum_{i=1}^{n} \mathbb{1}_{\left\{\chi \circ T^{i-1}>(1-\delta) \cdot g_{n}\right\}}<b_{n}\right) \\
& =\mu\left(\sum_{i=1}^{n} \overline{\mathbb{1}}_{\left\{\chi \circ T^{i-1}>(1-\delta) \cdot g_{n}\right\}}<b_{n}-n \cdot \mu\left(\chi>(1-\delta) \cdot g_{n}\right)\right) .
\end{aligned}
$$


By a calculation analogous to (19) and (20) we obtain that $n \cdot \mu\left(\chi>(1-\delta) \cdot g_{n}\right)>b_{n} /(1-\delta)^{\alpha} / 2$, for $n$ sufficiently large from which we can conclude

$$
\mu\left(\mathrm{T}_{n}^{(1-\delta) \cdot g_{n}} \chi>\mathrm{S}_{n}^{b_{n}} \chi\right) \leq \mu\left(\left|\sum_{i=1}^{n} \overline{\mathbb{1}}_{\left\{\chi \circ T^{i-1}>(1-\delta) \cdot g_{n}\right\}}\right|>\left((1-\delta)^{-\alpha / 2}-1\right) \cdot b_{n}\right),
$$

for $n$ sufficiently large. Applying Lemma 2.3 yields

$$
\begin{aligned}
\mu\left(\mathrm{T}_{n}^{(1-\delta) \cdot g_{n}} \chi>\mathrm{S}_{n}^{b_{n}} \chi\right) \leq K \cdot \exp \left(-U \cdot\left((1-\delta)^{-\alpha / 2}-1\right) \cdot \frac{b_{n}}{\left\|\overline{\mathbb{1}}_{\left\{\chi>(1-\delta) \cdot g_{n}\right\}}\right\|}\right. \\
\left.\cdot \min \left\{\left((1-\delta)^{-\alpha / 2}-1\right) \cdot \frac{b_{n}}{n \cdot\left|\overline{\mathbb{1}}_{\left\{\chi>(1-\delta) \cdot g_{n}\right\}}\right|_{1}}, 1\right\}\right),
\end{aligned}
$$

for $n$ sufficiently large.

Similarly as in (24) we obtain $\left|\overline{\mathbb{1}}_{\left\{\chi>(1-\delta) \cdot g_{n}\right\}}\right|_{1} \leq 4 \cdot(1-\delta)^{-\alpha / 2} \cdot b_{n} / n$, for $n$ sufficiently large and similarly as in (25) we obtain $\left\|\overline{\mathbb{1}}_{\left\{\chi>(1-\delta) \cdot g_{n}\right\}}\right\| \leq K_{1}+\|\mathbb{1}\|$. Setting

$$
A_{\alpha, \delta}:=\frac{U \cdot\left((1-\delta)^{-\alpha / 2}-1\right)}{K_{1}+\|\mathbb{1}\|} \cdot \min \left\{\frac{(1-\delta)^{\alpha / 2} \cdot\left((1-\delta)^{-\alpha / 2}-1\right)}{4}, 1\right\}
$$

implies

$$
\mu\left(\mathrm{T}_{n}^{(1-\delta) \cdot g_{n}} \chi>\mathrm{S}_{n}^{b_{n}} \chi\right) \leq K \cdot \exp \left(-A_{\alpha, \delta} \cdot b_{n}\right),
$$

for $n$ sufficiently large which tends to zero for $n$ tending to infinity.

Finally, we estimate $\mu\left(\mathrm{T}_{n}^{(1-\delta) \cdot g_{n}} \chi / d_{n}-1<-\epsilon\right)$. From (17) and (9) we can conclude that

$$
\int \mathrm{T}_{n}^{(1-\delta) \cdot g_{n}} \chi \mathrm{d} \mu \sim(1-\delta)^{\alpha} \cdot d_{n}
$$

Hence,

$$
\mu\left(\frac{\mathrm{T}_{n}^{(1-\delta) \cdot g_{n}} \chi}{d_{n}}-1<-\epsilon\right) \leq \mu\left(\overline{\mathrm{T}}_{n}^{(1-\delta) \cdot g_{n}} \chi<-\left(\epsilon+(1-\delta / 2)^{\alpha}-1\right) \cdot d_{n}\right)
$$

for $n$ sufficiently large. We can choose $\delta>0$ sufficiently small such that $\epsilon+(1-\delta / 2)^{\alpha}-1 \geq \epsilon / 2$ and an application of Lemma 2.3 yields

$$
\mu\left(\frac{\mathrm{T}_{n}^{(1-\delta) \cdot g_{n}} \chi}{d_{n}}-1<-\epsilon\right) \leq K \cdot \exp \left(-U \cdot \frac{\epsilon \cdot d_{n}}{2 \cdot\left\|^{(1-\delta) \cdot g_{n}} \bar{\chi}\right\|} \cdot \min \left\{\frac{\epsilon \cdot d_{n}}{\left.\left.2 \cdot n \cdot\right|^{(1-\delta) \cdot g_{n}} \bar{\chi}\right|_{1}}, 1\right\}\right)
$$

Similarly as above we have that $\left\|^{(1-\delta) \cdot g_{n}} \bar{\chi}\right\| \leq(1-\delta) \cdot g_{n} \cdot\left(K_{1}+\|\mathbb{1}\|\right)<g_{n} \cdot\left(K_{1}+\|\mathbb{1}\|\right)$ and $\left|{ }^{(1-\delta) \cdot g_{n}} \bar{\chi}\right|_{1} \leq\left.\left.\right|^{g_{n}} \bar{\chi}\right|_{1} \leq 3 d_{n}$ and with an analogous estimation as the one leading to (43) we obtain

$$
\mu\left(\frac{\mathrm{T}_{n}^{(1-\delta) \cdot g_{n}} \chi}{d_{n}}-1<-\epsilon\right) \leq K \cdot \exp \left(-\frac{U \cdot \epsilon^{2} \cdot(1-\alpha)}{13\left(K_{1}+\|\mathbb{1}\|\right) \cdot \alpha} \cdot b_{n}\right)
$$

for $n$ sufficiently large and $\epsilon$ sufficiently small, which tends to zero for $n$ tending to infinity.

\subsection{Proof of Theorem 1.10,}

Proof of Theorem 1.10. Since the Lebesgue measure is $T$-invariant, we notice that the system $\left([0,1), \mathcal{B}, T, \mu, B V,\|\cdot\|_{B V}, \chi\right)$ is precisely the system of piecewise expanding interval maps covered in [KS18, Section 1.4] with $B V$ defined as in Definition 1.8 The last condition to check is that there exists a constant $K_{1}$ such that for all $\ell>0$ we have that $\|\ell \chi\|_{B V} \leq K_{1} \cdot \ell$ and $\left\|\mathbb{1}_{\{\chi>\ell\}}\right\|_{B V} \leq K_{1}$ which is obviously fulfilled for our choice of $\chi$. 
Let $\omega \in[0, x)$ with $x \leq 2^{-b_{n}}$, then

$$
\chi(\omega)=\omega^{-\gamma},(\chi \circ T)(\omega)=\left(\omega \cdot 2^{-1}\right)^{-\gamma}, \ldots,\left(\chi \circ T^{b_{n}}\right)(\omega)=\left(\omega \cdot 2^{-b_{n}}\right)^{-\gamma}
$$

yielding $S_{n}^{b_{n}} \chi(\omega) \leq\left(\chi \circ T^{b_{n}}\right)(\omega)=\left(\omega \cdot 2^{-b_{n}}\right)^{-\gamma}$. Furthermore, $\left(\chi \circ T^{b_{n}}\right)(\omega)$ is decreasing in $\omega$. Hence, $\mu\left(S_{n}^{b_{n}} \chi \geq\left(\omega \cdot 2^{-b_{n}}\right)^{-\gamma}\right) \geq \omega$ and thus

$$
\int \mathrm{S}_{n}^{b_{n}} \chi \mathrm{d} \mu \geq \mu\left(\mathrm{S}_{n}^{b_{n}} \chi \geq\left(\omega \cdot 2^{-b_{n}}\right)^{-\gamma}\right) \cdot\left(\omega \cdot 2^{-b_{n}}\right)^{-\gamma} \geq \omega^{1-\gamma} \cdot 2^{-b_{n}} .
$$

Since $\omega$ can be chosen arbitrarily small, this implies $\int S_{n}^{b_{n}} \chi \mathrm{d} \mu=\infty$.

2.3. Proof of the statement in Remark 1.11. Last, we show how our results carry over to the i.i.d. case.

Proof of the statement in Remark 1.11, Let $\left(X_{n}\right)$ be a sequence of i.i.d. random variables mapping $\Omega \rightarrow \mathbb{R}$ with probability measure $\mathbb{P}$. Then we define $Y: \Omega \rightarrow \mathbb{R}^{\mathbb{N}}$ by $Y(\omega):=\left(X_{1}(\omega), X_{2}(\omega), \ldots\right)$. Further, let $\mu:=Y_{*} \mathbb{P}=\mathbb{P} \circ Y^{-1}$. Since the random variables $\left(X_{n}\right)$ are independent and identical distributed, $\mu$ can be written as $\mu=\mathbb{P} \circ X_{1}^{-1} \times \mathbb{P} \circ X_{2}^{-1} \times \ldots$ The measure $\mu$ is invariant and mixing with respect to the dynamics obtained by the shift map $\sigma: \mathbb{R}^{\mathbb{N}} \rightarrow \mathbb{R}^{\mathbb{N}}$ given by $\sigma\left(x_{1}, x_{2}, \ldots\right)=$ $\left(x_{2}, x_{3}, \ldots\right)$. If we write $x=x_{1} x_{2} \ldots$ and set $\chi(x)=x_{1}$, we obtain $\left(\chi \circ T^{n-1}\right) Y(\omega)=X_{n}(\omega)$.

Furthermore, we might introduce the Banach space of functions $\mathcal{F}$ on the shift space $\mathbb{R}^{\mathbb{N}}$ as all functions $f \in \mathcal{L}^{\infty}$ with $|\cdot|_{\infty}$ as a norm such that $f(x)$ is already determined by $x_{1}$. Obviously, $\mathcal{F}$ is a Banach space which contains the constant functions and fulfills (2) and (3).

Furthermore, the transfer operator $\widehat{T}$ of the transformation $T=\sigma$ has a spectral gap on $\mathcal{F}$. This can be easily seen by considering that for all $f \in \mathcal{L}^{1}$ and $g \in \mathcal{L}^{\infty}$ we have (11). In case that $f \in \mathcal{F}$ we even have that $\int \widehat{T} f \cdot g \mathrm{~d} \mu=\int f \mathrm{~d} \mu \cdot \int g \circ T \mathrm{~d} \mu$, which follows from the fact that $f$ and $g \circ T$ are independent with respect to $\mu$. If $\widehat{T} f=\int f \mathrm{~d} \mu$, the above equality is fulfilled for all $g \in \mathcal{L}^{\infty}$ and since the transfer operator is uniquely defined, the equality $\widehat{T} f=\int f \mathrm{~d} \mu$ has to hold. Since $\int f \mathrm{~d} \mu$ is a projection, we can write $\widehat{T} f=P f$ and do not even need an additional operator $N$, i.e. we have an even stronger statement than a spectral gap. It is also immediately clear that in the i.i.d. case we have $\boldsymbol{\psi}(n)=0$, for all $n \in \mathbb{N}$.

If we set $\chi(x)=x_{1}$ as the observable, then (4) are fulfilled and we can apply all theorems to this system.

\section{REFERENCES}

[Aar77] J. Aaronson. On the ergodic theory of non-integrable functions and infinite measure spaces. Israel J. Math., 27(2):163-173, 1977.

[AD01] J. Aaronson and M. Denker. Local limit theorems for partial sums of stationary sequences generated by Gibbs-Markov maps. Stoch. Dyn., 1(2):193-237, 2001.

[AN03] J. Aaronson and H. Nakada. Trimmed sums for non-negative, mixing stationary processes. Stochastic Process. Appl., 104(2):173-192, 2003.

[AN05] J. Aaronson and H. Nakada. On the mixing coefficients of piecewise monotonic maps. Israel J. Math., 148:1-10, 2005.

[AZ14] J. Aaronson and R. Zweimüller. Limit theory for some positive stationary processes with infinite mean. Ann. Inst. Henri Poincaré Probab. Stat., 50(1):256-284, 2014.

[BGT87] N. H. Bingham, C. M. Goldie, and J. L. Teugels. Regular Variation. Cambridge University Press, Cambridge, 1987.

[Bra05] R. C. Bradley. Basic properties of strong mixing conditions. A survey and some open questions. Probab. Surv., 2:107-144, 2005.

[Dou94] P. Doukhan. Mixing: Properties and Examples. Springer, New York, 1994.

[DV86] H. G. Diamond and J. D. Vaaler. Estimates for partial sums of continued fraction partial quotients. Pacific Journal of Mathematics, 122(1):73-82, 1986.

[Fel71] W. Feller. An introduction to probability theory and its applications II. Wiley, New York, 2 edition, 1971. 
[FJ03] A. Fan and Y. Jiang. Spectral theory of transfer operators. In Complex dynamics and related topics: lectures from the Morningside Center of Mathematics, volume 5 of New Stud. Adv. Math., pages 63-128. Int. Press, Somerville, MA, 2003.

[Gou10] S. Gouëzel. Characterization of weak convergence of Birkhoff sums for Gibbs-Markov maps. Israel J. Math., 180:1-41, 2010.

[Gou15] S. Gouëzel. Limit theorems in dynamical systems using the spectral method. In Hyperbolic dynamics, fluctuations and large deviations, volume 89 of Proc. Sympos. Pure Math., pages 161-193. Amer. Math. Soc., Providence, RI, 2015.

[Hae93] E. Haeusler. A nonstandard law of the iterated logarithm for trimmed sums. Ann. Probab., 21(2):831-860, 1993.

[Hay14] A. Haynes. Quantitative ergodic theorems for weakly integrable functions. Ergodic Theory Dynam. Systems, 34(2):534-542, 2014.

[HM87] E. Haeusler and D. M. Mason. Laws of the iterated logarithm for sums of the middle portion of the sample. Math. Proc. Cambridge Philos. Soc., 101(02):301-312, 1987.

[Kes93] H. Kesten. Convergence in distribution of lightly trimmed and untrimmed sums are equivalent. Mathematical Proceedings of the Cambridge Philosophical Society, 113(3):615-638, 1993.

[KMS16] M. Kesseböhmer, S. Munday, and B. O. Stratmann. Infinite ergodic theory of numbers. De Gruyter Graduate. De Gruyter, Berlin, 2016.

[KS17] M. Kesseböhmer and T. Schindler. Strong laws of large numbers for intermediately trimmed sums of i.i.d. random variables with infinite mean. J. Theoret. Probab., pages 1-19, 2017.

[KS18] M. Kesseböhmer and T. Schindler. Strong laws of large numbers for intermediately trimmed Birkhoff sums of observables with infinite mean. Stochastic Process. Appl. to appear, pages 1-45, 2018.

[KS19] M. Kesseböhmer and T. Schindler. Intermediately trimmed strong laws for Birkhoff sums on subshifts of finite type. preprint: arXiv:1901.04478, 2019.

[LY73] A. Lasota and J. A. Yorke. On the existence of invariant measures for piecewise monotonic transformations. Trans. Amer. Math. Soc., 186:481-488 (1974), 1973.

[Mor76] T. Mori. The strong law of large numbers when extreme terms are excluded from sums. Z. Wahrsch. Verw. Gebiete, 36(3):189-194, 1976.

[Mor77] T. Mori. Stability for sums of i.i.d. random variables when extreme terms are excluded. Z. Wahrsch. Verw. Gebiete, 40(2):159-167, 1977.

[MZ15] I. Melbourne and R. Zweimüller. Weak convergence to stable Lévy processes for nonuniformly hyperbolic dynamical systems. Ann. Inst. Henri Poincaré Probab. Stat., 51(2):545-556, 2015.

[Pra60] J. W. Pratt. On interchanging limits and integrals. Ann. Math. Statist., 31:74-77, 1960.

[Ryc83] M. Rychlik. Bounded variation and invariant measures. Studia Math., 76(1):69-80, 1983.

[Sar06] O. Sarig. Continuous phase transitions for dynamical systems. Comm. Math. Phys., 267(3):631-667, 2006.

[TK10] M. Tyran-Kamińska. Weak convergence to Lévy stable processes in dynamical systems. Stoch. Dyn., 10(2):263-289, 2010.

[Zwe98] R. Zweimüller. Ergodic structure and invariant densities of non-Markovian interval maps with indifferent fixed points. Nonlinearity, 11(5):1263-1276, 1998.

Universität Bremen, Fachbereich 3 - Mathematik und Informatik, Bibliothekstr. 1, 28359 Bremen, GERMANy

E-mail address: mhk@math.uni-bremen.de

Australian National University, Research School of Finance, Actuarial Studies and Statistics, 26C Kingsley St, Acton ACT 2601, Australia

E-mail address: tanja.schindler@anu.edu.au 